\title{
Analysis of Angular Threshold Criteria for Transient Instability Identification in Uncertain Power Systems
}

\author{
Juan D. Morales \\ Jovica V. Milanović \\ School of Electrical and Electronic Engineering \\ The University of Manchester \\ Manchester, UK \\ juan.morales@manchester.ac.uk \\ jovica.milanovic@manchester.ac.uk
}

\author{
Panagiotis N. Papadopoulos \\ Department of Electronic and Electrical Engineering \\ University of Strathclyde \\ Glasgow, UK \\ panagiotis.papadopoulos@strath.ac.uk
}

\begin{abstract}
This paper addresses the problem of finding a threshold (critical value of rotor angle) for the identification of transient instability by the sole use of the information on rotor angles. Several threshold values for rotor angles have been used in the past without a clear agreement on a standard value. In this paper a probabilistic approach to the problem is proposed, so that for any network a threshold can be defined based on set accuracy, while at the same time minimizing the instability identification time. Results demonstrate that highly accurate robust rotor angle thresholds can be found, even for highly uncertain power networks with a high penetration of Renewable Energy Sources (RES).
\end{abstract}

Index Terms-Center of inertia, transient instability identification, transient instability threshold, uncertainties.

\section{INTRODUCTION}

Ensuring the stability of power networks is a continuing concern among system operators all around the world. Stricter and more uncertain operation conditions due to market based rules and a growing integration of Renewable Energy Sources (RES), respectively, are becoming typical features of modern power systems that are not likely to change in the foreseeable future, and this could affect their transient stability negatively. The mitigation of potential stability problems can be achieved either by the application of preventive or corrective control.

Preventive control measures are mainly comprised of generation rescheduling actions taken in advance to avoid the loss of synchronism after reasonable expected contingencies. The general idea is to do the rescheduling in the most optimal, or nearly optimal, way possible. This is typically done by following rigorous optimization procedures for developing Transient Stability Constrained Optimal Power Flow (TSCOPF) techniques [1-5], or by the aid of Artificial Intelligence (AI) algorithms [6-8] for finding an optimal re-dispatch of generators such that transient security is guaranteed.

In the case of corrective or emergency control, planned automatic measures are taken after a disturbance in order to avoid instability. These are typically the actuation of

This work was supported by the School of Electrical and Electronic Engineering PhD scholarship award of The University of Manchester and by the EPSRC, U.K., through the HubNet consortium (grant number: $\mathrm{EP} / \mathrm{N} 030028 / 1)$. strategically deployed devices or the application of disruptive measures such as generation/load shedding and islanding schemes, among others [9-11]. The increasing availability of Phasor Measurement Units (PMUs) throughout power systems has increased the research interest in online real time corrective control for transient stability improvement that can effectively use such information for triggering corrective actions [12-15]. In consequence, predictive techniques capable of assessing whether a network disturbance will evolve into a stable or unstable condition in advance by means of processing PMU information have been the focus of numerous research works in the past years, e.g. [15-17].

The key feature for the success in all the aforementioned applications of preventive and corrective control, is the transient stability criterion chosen for the accurate and timely identification of instability. A review of different existing methods for this can be found in e.g., $[12,18]$. One of the most straightforward of such methods is the angle threshold criterion. It is based on the principle that there is a limit in the value that the generator rotor angles can diverge (separate) from each other or with respect to a predefined angle reference. If this limit/threshold is exceeded, then synchronism cannot be maintained anymore and the system loses stability. Although very simple in concept, the disadvantage of this approach is that for multi-machine systems there is no way to find analytically their exact stability boundaries [18, 19], and therefore there is not a fixed value for the angle threshold for the instability occurrence. The boundary in angle terms, is usually defined for each system separately using engineering heuristic criteria as even for one system the threshold might change due to the many different operating conditions the network is subjected to [13]. Hence several threshold values have been used in the past. In addition, increasing penetration of RES and thus an increase in uncertainty in system operation raises the question of the extent to which the variability of possible transient instability thresholds might be affected.

This paper addresses some of the drawbacks of the angle threshold method for transient instability identification so that the inherent heuristics usually involved in the definition of the 
instability angle thresholds are reduced. The main objectives of this study were : i) To clarify the concepts related to the use of angle thresholds for the identification of instability; ii) To present a probabilistic approach to the problem, so that the threshold can be defined based on a required accuracy for transient instability identification. This will allow an estimation of the angle thresholds as well as the variability of threshold values in a realistic large network subjected to a wide range of different operating conditions and to a wide range of RES penetration levels; iii) To assess how much the instability identification can be sped up using lower angle threshold values without significantly reducing the accuracy of instability identification. This information will facilitate faster identification of potential instability and faster deployment of corrective control measures.

\section{TRANSIENT INSTABILITY IDENTIFICATION BY MEANS OF AN ANGLE THRESHOLD}

\section{A. Instability in a One Machine Infinite Bus (OMIB) System}

To introduce the main concepts of the instability identification, the well-known analysis for an OMIB system is presented here. The customary assumption for this analysis is the use of the classical model for the synchronous generator representation (voltage source behind a transient reactance, both constant), neglecting speed governor and other controls, in addition to all the resistive parameters of the network.

Fig. 1 shows the power - angle curve that can be constructed for the stability analysis in this case, the actual rotor angle of the only generator in the network can be monitored, and measured with respect to the fixed reference angle of the infinite bus. Using the Equal Area Criterion (EAC) principle, it is observed that the maximum excursion angle $\delta_{L I M}$ for any condition where stability can be maintained $(\mathrm{A} 1=\mathrm{A} 2)$, will always be a value below $180^{\circ}$. Then it can be said that all rotor angle swings that reach a value of $180^{\circ}$ can be deemed to be unstable with a $100 \%$ of probability. The exact $\delta_{\text {LIM }}$ angle beyond which stability cannot be maintained will always be below the threshold of $180^{\circ}$ but it will depend on the exact operating conditions of the network, mostly influenced by the delivered mechanical power, network loading, voltage, and the fault type. Therefore, a threshold of $180^{\circ}$ can be considered to be robust but over conservative.

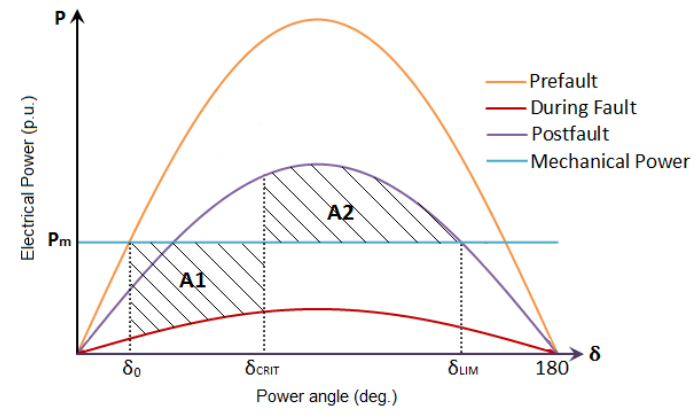

Figure 1. Power - angle curve for an OMIB system showing the EAC

\section{B. Instability in Real Multi-Machine Networks}

In real systems, none of the assumptions made for the classical stability analysis of an OMIB system hold firm. The maximum rotor oscillations before instability happens will be strongly influenced by the much more complex real behavior of generators' dynamics and their controllers' interaction, which are always tuned to increase network stability. Hence in real systems, strong rotor oscillations could reach larger magnitudes before actually losing synchronism, compared to an OMIB system. In addition, no infinite bus exists, and the rotor angles are measured as relative angles with respect to a pre-defined reference, which can be either the rotor angle of one chosen synchronous generator or the angle of the Center Of Inertia (COI) of the system [20].

Hence the task of identifying the exact point of instability becomes more difficult by comparison with the situation of having an infinite bus with a reference angle rotating at invariable synchronous speed. The reference chosen for measuring the relative rotor angles will therefore have a direct influence on the thresholds set for the detection of instability.

\section{Threshlolds Based on the Maximum Angle Difference Between any Two Generators}

For multi-machine systems, the instability phenomenon is manifested as the separation of a generator or group of generators with respect to the rest, until they cannot maintain synchronism and start shifting away from each other indefinitely. The angle threshold for instability identification $\delta_{T}$ represents the maximum angular distance that can be held between two rotor angles without losing synchronism, hence instability is reached if the condition defined by (1) is fulfilled.

$$
\delta_{T}<\delta_{\text {MAX }}=\max \left(\left|\delta_{i}-\delta_{j}\right|\right)
$$

where $\delta_{M A X}$ is the maximum angle difference (electrical degrees) between any two rotor angles $\delta_{i}$ and $\delta_{j}$ for the same instant of time after the occurrence of a large disturbance.

In the OMIB system analyzed in Section II-A, it was established that for a $180^{\circ}$ angle threshold a loss of synchronism will have definitely occurred. For the multimachine system, several rotor angles oscillate at the same time with respect to each other, and as established in Section II-B, it is expected that strong oscillations might reach high values without a loss of synchronism. Hence, it is not expected that a $180^{\circ}$ angle separation between two generators will definitely lead to a condition of instability or at least not in $100 \%$ of cases $[21,22]$. Studies that have used the $180^{\circ}$ threshold in the past include [5, 19, 23-26].

On the other hand, a threshold of $360^{\circ}$ means that two generators are one complete cycle away from each other after a disturbance, and it is expected that for this point instability has definitely occurred. Studies reporting the use of this threshold include [6, 16, 21, 22, 27-33]. References [21, 22] that investigated thresholds by conducting an extensive number of simulations for large systems found that instability actually occurs at thresholds lower than $360^{\circ}$, and also that these could be below and above $180^{\circ}$. It can be concluded therefore that the $360^{\circ}$ threshold is the safest option for identifying instability, however, it will require more time for identification compared to lower thresholds.

\section{Thresholds Based on Angular Distance from the COI}

The COI is a concept widely used for the stability analysis of multi-machine power systems. The angular position $\delta_{C O I}$ of 
the COI, is defined as the inertia weighted average of all rotor angles, see (2), and it represents the "mean motion" of the system [20]. The speed $\omega_{\mathrm{COI}}$ of the COI is defined by (3).

$$
\begin{gathered}
\delta_{C O I}=\frac{\sum_{i=1}^{n} H_{i} \times \delta_{i}}{\sum_{i=1}^{n} H_{i}} \\
\omega_{C O I}=\frac{\sum_{i=1}^{n} H_{i} \times \omega_{i}}{\sum_{i=1}^{n} H_{i}}
\end{gathered}
$$

where $H_{i}$ is the inertia constant $(\mathrm{s}), \delta_{i}$ is the rotor angle (electrical degrees) and $\omega_{i}$ is the rotor angular speed (p.u.) of generator $i$, while $n$ is the total number of generators.

The COI idea was first introduced in [34] as an analogous concept to the center of mass for mechanical systems, in order to have a way of decomposing the network dynamics into relative and collective motions, such that a distinction between synchronous and frequency equilibrium could be established. Since the time derivative of the COI angular position represents an accurate estimate of the entire system's frequency, it can be used to assess its frequency equilibrium. On the other hand, by measuring the rotor angles with respect to the COI, the synchronous equilibrium of the system can also be distinctively assessed.

The oscillations and speed of the COI are much more stationary (slower) compared to those corresponding to each generator, since the total inertia of the system $H_{T}=\sum_{i=1}^{n} H_{i}$ is much greater than the individual inertias of each generator. Therefore, it can be said that if the COI is defined as the reference for angle measurement, its behavior is comparable to a fixed reference like the one provided by the infinite bus in the OMIB system analyzed in Section II-A, and the stability analysis can be performed in a similar way. This means that an oscillation that reaches a $180^{\circ}$ difference from the COI would guarantee that instability has occurred. Formally, when using the COI reference frame, instability is reached if condition (4) is fulfilled at some point in time after a large disturbance.

$$
\delta_{T-\mathrm{COI}}<\left|\delta_{i}-\delta_{\mathrm{COI}}\right|
$$

where $\delta_{T-C O I}$ is the transient instability threshold in the COI frame. In past work, e.g. $[1-5,12-15,22]$, the value $\delta_{T-C O I}$ is indistinctively defined in the range of $100^{\circ}-180^{\circ}$.

Condition (4) can be applied to any oscillation pattern since the focus is only to guarantee that instability is reached. Further information regarding system separation into dynamically coherent areas could also be obtained using the COI concept for measuring angles and information from the rotor speeds with respect to the COI using (3). However, this is out of the scope of the present paper.

\section{PROBABILISTIC FRAMEWORK FOR DETERMINING THE ANGLE THRESHOLD FOR DETECTION OF INSTABILITY}

This paper proposes that the threshold value for instability identification is defined by choosing a minimum acceptable value that guarantees a required accuracy for the identification of instability, while at the same time minimizing the heuristics involved in its determination and avoiding extremely conservative values, as discussed in previous sections. The accuracies can be obtained from the calculation of the probability of instability if a certain angle threshold is reached during a post-fault oscillation, as defined in Section IV-B. The Monte Carlo (MC) based methodology used in this paper for obtaining a database of representative operating conditions and system dynamic angular responses for the probabilities to be calculated, is illustrated in Fig. 2. It is adapted from [33] and previously applied by the authors in [30]. This approach is suited to the Transient Stability Assessment (TSA) of modern uncertain power networks with the inclusion of RES, reflecting thoroughly their statistical dynamic behavior.

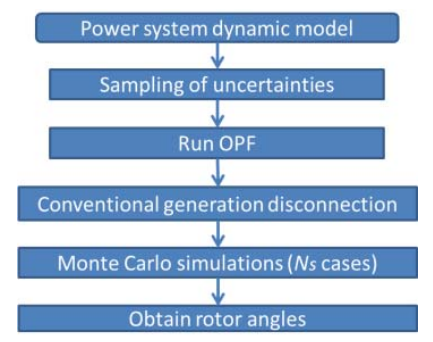

Figure 2. Proposed TSA methodology (adapted from [33])

\section{A. Test Network and Modelling Criteria}

The test network used in this paper shown in Fig. 3 is the modified IEEE 68 bus - 16 machine test network with the inclusion of 10 RES plants. All generators are represented by full sixth order models including AVR and governor controls, plus a PSS for G9. The system loads are modelled as constant impedances. The RES part of the network consists of a mix of Doubly Fed Induction Generators (DFIGs), representing wind generators and Full Converter Connected (FCC) units, representing wind generators and PV units, all of them with Fault Ride Through (FRT) capabilities. Complete data of the system components and models used can be found in $[30,33]$.

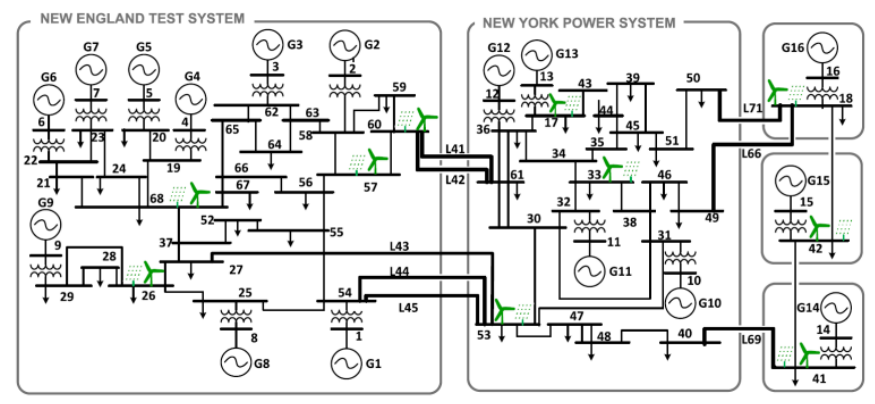

Figure 3. Modified IEEE 68 bus test network with the inclusion of RES

\section{B. Modelling of Uncertainties}

The sampling of uncertainties is done separately according to appropriate probability distributions describing the particular behavior for the following parameters: system load, hour of the day, wind/PV production, fault duration and fault location. This approach guarantees that a representative range of possible operating conditions and fault locations are covered for the analysis in a probabilistic way. Only three phase self-clearing faults are considered with a mean value of 14 cycles and a standard deviation of $6.67 \%$ to model their duration, which is selected to generate an appropriate number of stable/unstable cases for analysis. Complete information about the sampling of uncertainties can be found in $[30,33]$. 
Pre-fault conditions are obtained by solving an Optimal Power Flow (OPF), and considering a fixed 15\% amount of spinning reserve for all cases, unless it is not feasible due to high load conditions, in which case the reserve is reduced accordingly. The OPF dispatch determines the amount of conventional generation disconnection and corresponding system inertia reduction due to RES penetration. Complete information on pre-fault conditions can be found in $[30,33]$.

\section{Monte Carlo Simulations}

A number of MC simulations, $N_{S}$, are performed for each scenario after considering all uncertainties. The required number of simulations defined in [33] to achieve sufficient accuracy was 6000 , in this study as in [30], the number of simulations is increased to 10000 in order to obtain more unstable cases for credible statistical analysis.

A total of 11 scenarios are generated with RES penetration levels varying between $0-90 \%$ in $10 \%$ steps, plus an additional step with a RES penetration level of $55 \%$ (since a turning point in terms of impact of RES to system stability around that point has been observed). The amount of installed capacity of RES per scenario is given as a percentage of the total installed conventional generation capacity of the system (in MW). The actual amount of power produced by RES generation will depend on the resulting OPF considering system uncertainties and not on the nominal penetration level. Each MC simulation involves a post-fault Time Domain Simulation (TDS) set to 5 $\mathrm{s}$ with the corresponding three-phase fault applied at $1 \mathrm{~s}$, since the analysis focuses on first swing transient instability. All simulations are performed in the DigSILENT - Power Factory software [35].

\section{Results FOR THE ANALYSIS IN THE TEST System}

\section{A. Number of Instabilities with Different Thresholds}

Fig. 4 shows the total number of unstable cases for each scenario identified with the most common thresholds for instability detection used in literature. The only aspect to be concerned with is the accuracy of instability identification, since the resulting patterns obtained are the same for all thresholds, i.e. they follow accurately the behavior of the system. The behavior in this case means that as the penetration level increases, the number of unstable cases is reducing until the $55-60 \%$ RES penetration level, after which the number of unstable cases starts to increase. As expected, the lower the threshold used, the higher the number of identified unstable cases. Particularly for the $\delta_{T-C O I}$ threshold of $100^{\circ}$, the number of identified instabilities is quite high compared to the most conservative $\delta_{T}$ of $360^{\circ}$ or even the $\delta_{T-C O I}$ threshold of $150^{\circ}$.

\section{B. Cumulative Distribution Functions (CDFs) for the Probability of Instability Identification}

The accuracy of instability identification using different thresholds is derived from the CDFs shown in Fig. 5 and Fig. 6 , in which the Probability of Instability (P.I.) when an angle threshold is reached is calculated according to eq. (5).

$$
P . I .=\frac{A . N \cdot I .}{N \cdot I \cdot \delta_{T}}
$$

where A.N.I. is the actual number of instabilities per scenario, and $N . I \cdot \delta_{T}$ the number of instabilities identified per scenario using a variable threshold $\delta_{T}$ (or $\delta_{T-C O I}$ ). The A.N.I. is calculated for all cases using the base $\delta_{T}$ threshold of $360^{\circ}$, since it was established in previous sections that it guarantees the identification of instability with a $100 \%$ of accuracy.

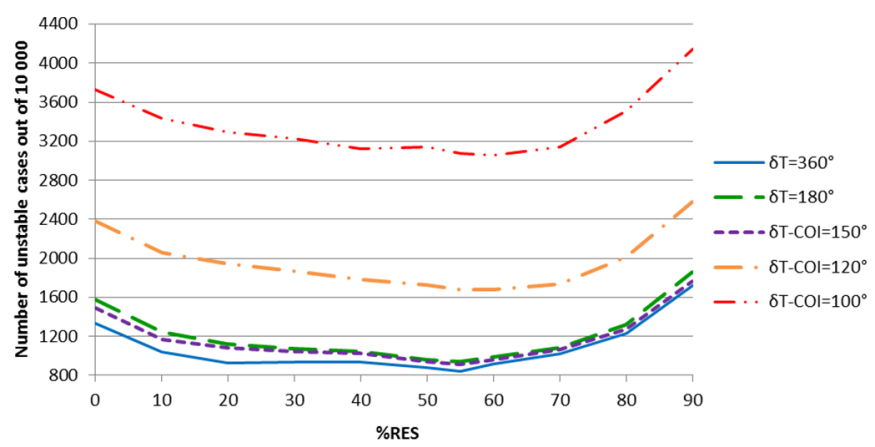

Figure 4. Total number of unstable cases out of 10000 for five thresholds

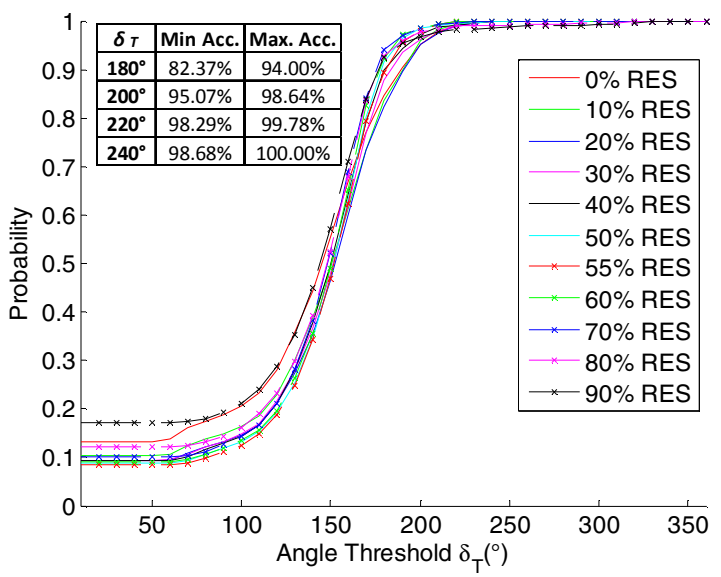

Figure 5. CDF of the P.I for different $\delta_{T}$ values

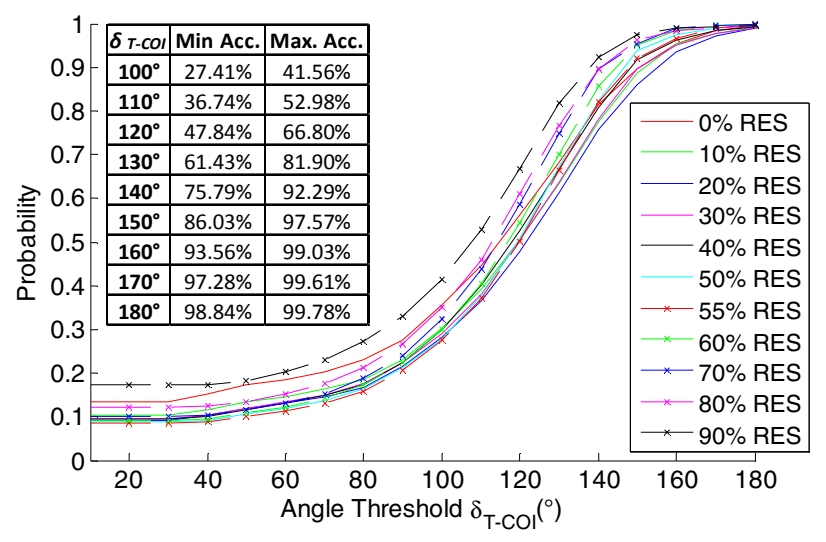

Figure 6. $\mathrm{CDF}$ of the P.I. for different $\delta_{T-\mathrm{COI}}$ values

With respect to the $\delta_{T}$ thresholds in Fig. 5, it can be seen that the $180^{\circ}$ threshold does not guarantee full confidence in the accurate identification of instability, as previously discussed, providing confidence levels in the range of $82-94 \%$, i.e., the probability that the instability is identified correctly with this threshold varies from $82 \%$ to $94 \%$ depending on the case study (penetration level of RES). For a $200^{\circ}$ threshold, the probability that the instability is identified correctly varies from $95-99 \%$, and $99-100 \%$ for a $240^{\circ}$ threshold. 
With respect to the $\delta_{T-C O I}$ thresholds in Fig. 6, the commonly used threshold value of $100^{\circ}$ results in a relatively low probability that the instability is identified correctly, i.e., $27-42 \%$, although it improves to $48-67 \%$ for a threshold of $120^{\circ}$ which is also commonly used in literature. Upper thresholds result in high accurate values for the probability of instability identification, varying from $86-98 \%$ for $150^{\circ}$ to 99 $100 \%$ for $180^{\circ}$. Results show that only the $\delta_{T-C O I}$ threshold of $180^{\circ}$ guarantees practically a $100 \%$ of accuracy, making the instability identification of a multi-machine system and that of an OMIB system resemble conceptually and practically, as expected and previously discussed in Section II-D.

When using higher threshold values it can be observed that: i) the accuracy of the identification of instability is increased (as expected); ii) the range of variation of accuracy obtained for the different scenarios analyzed is considerably reduced. Therefore, neither the number of operating conditions nor the inertia reduction (due to the penetration of RES) have a strong influence on the accuracy of assessment when using high values of angle thresholds for instability identification. It can be concluded therefore, that a unique highly accurate threshold could be chosen for a large uncertain system and used for accurate instability identification.

\section{Estimated Reduction in Time for Instability Identification}

Fig. 7 shows the Probability Density Functions (PDFs) of the time to instability obtained with the $\delta_{T}$ base threshold of $360^{\circ}$ and also the main statistical parameters for each PDF. Fig. 8 and Fig. 9 show the PDFs of the reduction in time for instability identification for the $99 \%$ accurate thresholds $\delta_{T}$ of $240^{\circ}$ and $\delta_{T-C O I}$ of $180^{\circ}$, with respect to the base case observed in Fig. 7. The accuracy for instability identification and the statistical parameters for each PDF or scenario are also shown. It can be seen that both thresholds yield considerable reductions in the times to instability, although the $\delta_{T-C O I}$ threshold of $180^{\circ}$ produces larger reductions in the times to instability identification (190-210 ms mode) compared to the $\delta_{T}$ threshold of $240^{\circ}$ (130-140 ms mode) with practically the same probability that the instability is identified correctly for all penetration scenarios. Lower angle threshold values would yield greater time reductions but lower accuracies.

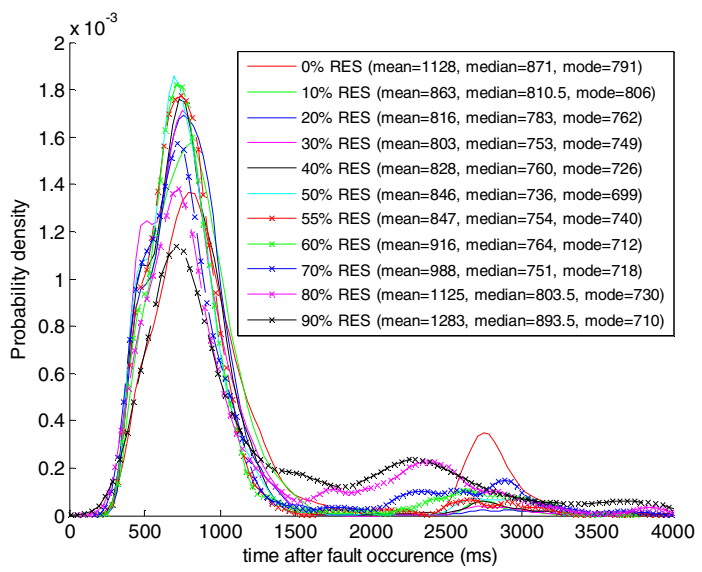

Figure 7. PDF time to instability using the base threhsold $\delta_{T}=360^{\circ}$

It can be observed in Fig. 9, mostly for the $0 \%$ RES level, that a small portion of the PDFs extends to negative times, which means that the instability identification is actually delayed when using thresholds in the COI frame for some cases. This happens mostly for oscillations that are second or further swing unstable.

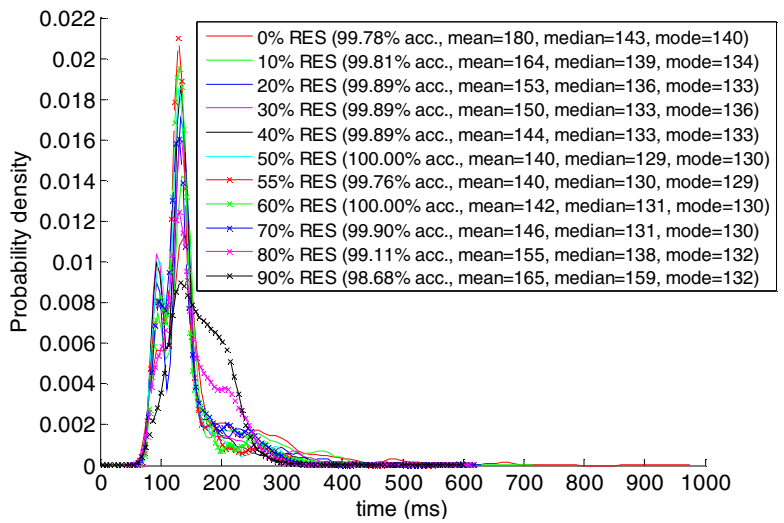

Figure 8. PDFs time reduction in instability identification with $\delta T=240^{\circ}$

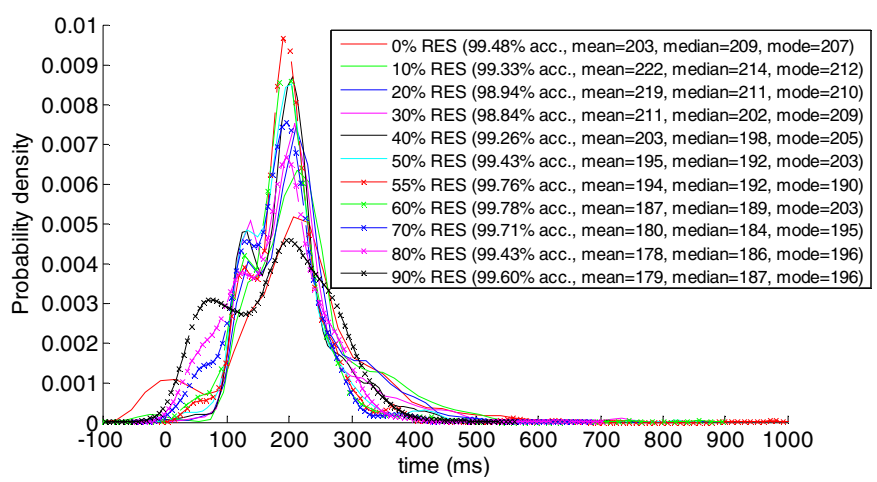

Figure 9. PDFs time reduction in instability identification with $\delta_{T-C O I}=180^{\circ}$

\section{CONCLUSIONS}

This paper discussed several concepts related to the identification of transient instability solely by means of processing recorded rotor angle information. It was found that the COI frame offers some advantages conceptually for the identification of instability in multi-machine systems, and that high conservative threshold values (large values of rotor angles) should be used in spite of the longer processing times required for instability identification.

By the application of a probabilistic approach, an appropriate sufficiently accurate threshold for instability identification can be found, without the need to use conservative values, or relying highly on heuristic engineering criteria. The probabilistically obtained realistic angle responses of a large system with a wide range of RES penetration levels were used to calculate the probability of an accurate identification of instability with different thresholds. It was found that the identified thresholds are robust and that even for highly uncertain networks, a unique and accurate non conservative angle threshold could be defined.

A threshold of $240^{\circ}$ when assessing instability by means of the maximum rotor angle difference between two generators and a threshold of $180^{\circ}$ when using the COI reference frame were found to have very high accuracy (99\%) of instability 
identification for the test network analyzed, with the advantage of providing considerable reductions in the time of instability identification $(130-210 \mathrm{~ms})$ with respect to the defined base threshold used as a benchmark. The COI frame threshold provided larger reductions, i.e. a possibly faster identification of instability. Since the analysis was performed using a reasonably large test network, it is reasonable to assume that both defined thresholds would also be highly accurate for use in any realistic large network, if detailed analysis is not wanted or possible. Since it is conceptually more appealing and was also found to provide larger reductions in the instability identification time, the use of the COI frame based thresholds represents a better option for transient instability identification.

\section{REFERENCES}

[1] D. Gan, R. J. Thomas, and R. D. Zimmerman, "Stability-constrained optimal power flow," IEEE Transactions on Power Systems, vol. 15, no. 2, pp. 535-540, 2000.

[2] L. Chen, Y. Taka, H. Okamoto, R. Tanabe, and A. Ono, "Optimal operation solutions of power systems with transient stability constraints," IEEE Trans. on Circuits and Systems I: Fundamental Theory and Applications, vol. 48, no. 3, pp. 327-339, 2001.

[3] Y. Xia and K. W. Chan, "Dynamic Constrained Optimal Power Flow Using Semi-Infinite Programming," IEEE Transactions on Power Systems, vol. 21, no. 3, pp. 1455-1457, 2006.

[4] Q. Jiang and Z. Huang, "An Enhanced Numerical Discretization Method for Transient Stability Constrained Optimal Power Flow," IEEE Trans. on Power Systems, vol. 25, no. 4, pp. 1790-1797, 2010.

[5] X. Huanhai, G. Deqiang, H. Zhilong, Z. Kanqin, and C. Lu, "Applications of Stability-Constrained Optimal Power Flow in the East China System," IEEE Transactions on Power Systems, vol. 25, no. 3, pp. 1423-1433, 2010.

[6] C. F. Kucuktezcan and V. M. I. Genc, "Big bang-big crunch based optimal preventive control action on power systems," in $20123 \mathrm{rd}$ IEEE PES Innovative Smart Grid Technologies Europe (ISGT Europe), 2012, pp. 1-4.

[7] C.-J. Ye and M.-X. Huang, "Multi-Objective Optimal Power Flow Considering Transient Stability Based on Parallel NSGA-II," IEEE Transactions on Power Systems, vol. 30, no. 2, pp. 857-866, 2015.

[8] N. Mo, Z. Y. Zou, K. W. Chan, and T. Y. G. Pong, "Transient stability constrained optimal power flow using particle swarm optimisation," IET Generation, Transmission \& Distribution, vol. 1, no. 3, 2007.

[9] D. Ruiz-Vega and M. Pavella, "A comprehensive approach to transient stability control. II. Open loop emergency control," IEEE Transactions on Power Systems, vol. 18, no. 4, pp. 1454-1460, 2003.

[10] J. D. Morales, P. N. Papadopoulos, and J. V. Milanović, "Feasibility of different corrective control options for the improvement of transient stability," in 2017 IEEE Manchester PowerTech, 2017, pp. 1-6.

[11] A. Kyriacou, P. Demetriou, C. Panayiotou, and E. Kyriakides, "Controlled Islanding Solution for Large-Scale Power Systems," IEEE Transactions on Power Systems, vol. 33, no. 2, pp. 1591-1602, 2018.

[12] X. Wu, A. Xu, J. Zhao, H. Deng, and P. Xu, "Review on transient stability prediction methods based on real time wide-area phasor measurements," in 2011 4th International Conference on Electric Utility Deregulation and Restructuring and Power Technologies (DRPT), 2011, pp. 320-326.

[13] M. Sherwood, H. Dongchen, and V. M. Venkatasubramanian, "Realtime detection of angle instability using synchrophasors and action principle," in 2007 iREP Symposium - Bulk Power System Dynamics and Control - VII. Revitalizing Operational Reliability, 2007, pp. 1-11.

[14] G. V. N. YatendraBabu, N. R. Naguru, and V. Sarkar, "Real-time transient instability detection using frequency trend vector in wide area monitoring system," in 2016 IEEE 6th International Conference on Power Systems (ICPS), 2016, pp. 1-4.

[15] S. A. Siddiqui, K. Verma, K. R. Niazi, and M. Fozdar, "Artificial neural network based early detection of real-time transient instability for initiation of emergency control through wide-area synchrophasor measurements," in 2015 International Conference on Computer, Communication and Control (IC4), 2015, pp. 1-6.

[16] T. Guo and J. V. Milanovic, "Online Identification of Power System Dynamic Signature Using PMU Measurements and Data Mining," IEEE Trans. on Power Systems, vol. 31, no. 3, pp. 1760-1768, 2016.

[17] P. N. Papadopoulos, T. Guo, and J. V. Milanović, "Probabilistic Framework for Online Identification of Dynamic Behavior of Power Systems With Renewable Generation," IEEE Transactions on Power Systems, vol. 33, no. 1, pp. 45-54, 2018.

[18] H. Xin, D. Can, Y. Li, T. S. Chung, and J. Qiu, "Transient stability preventive control and optimization via power system stability region analysis," in 2006 IEEE Power Engineering Society General Meeting, 2006, p. 8 pp.

[19] D. Gan, Z. Qu, and X. Wu, "Loadability of power systems with steadystate and dynamic security constraints," International Journal of Electrical Power and Energy Systems, Article vol. 25, no. 2, pp. 91-96, 2003.

[20] P. W. Sauer, M. A. Pai, and J. H. Chow, Power System Dynamics and Stability. Hoboken, NJ, USA: John Wiley \& Sons, 2018.

[21] S. M. Rovnyak, M. N. Nilchi, D. W. Longbottom, and D. C. Vasquez, "Angle stability predictive indices," in 2012 IEEE Power and Energy Society General Meeting, 2012, pp. 1-6.

[22] R. Lyu et al., "The qualitative criterion of transient angle stability," in 10th International Conference on Advances in Power System Control, Operation \& Management (APSCOM 2015), 2015, pp. 1-6.

[23] D. Gan, D. E. B. Chattopadhyay, and X. Luo, "Enhancements to Stability Constrained OPF to Overcome Sub-optimality," Electric Power Components and Systems, vol. 33, no. 5, pp. 481-491, 2005.

[24] L. Chih-Wen and J. S. Thorp, "New methods for computing power system dynamic response for real-time transient stability prediction," IEEE Transactions on Circuits and Systems I: Fundamental Theory and Applications, vol. 47, no. 3, pp. 324-337, 2000

[25] C. Liu and J. Thorp, "Application of synchronised phasor measurements to real-time transient stability prediction," IEE Proceedings - Generation, Transmission and Distribution, vol. 142, no. 4, pp. 355-360, 1995.

[26] S. Rovnyak, L. Chih-Wen, L. Jin, M. Weimin, and J. Thorp, "Predicting future behavior of transient events rapidly enough to evaluate remedial control options in real-time," IEEE Transactions on Power Systems, vol. 10, no. 3, pp. 1195-1203, 1995.

[27] S. Rovnyak, S. Kretsinger, J. Thorp, and D. Brown, "Decision trees for real-time transient stability prediction," IEEE Transactions on Power Systems, vol. 9, no. 3, pp. 1417-1426, 1994.

[28] I. Genc, R. Diao, V. Vittal, S. Kolluri, and S. Mandal, "Decision TreeBased Preventive and Corrective Control Applications for Dynamic Security Enhancement in Power Systems," IEEE Transactions on Power Systems, vol. 25, no. 3, pp. 1611-1619, 2010.

[29] C. Liu et al., "A Systematic Approach for Dynamic Security Assessment and the Corresponding Preventive Control Scheme Based on Decision Trees," IEEE Transactions on Power Systems, vol. 29, no. 2, pp. 717-730, 2014.

[30] J. D. Morales, P. N. Papadopoulos, and J. V. Milanović, "Statistical Assessment of the Impact of Renewable Energy Sources on Transient Stability," in 2018 IEEE PES Innovative Smart Grid Technologies Conference Europe (ISGT-Europe), 2018, pp. 1-6.

[31] DSA TOOLS TSAT User Manual. Powertech Labs Inc., Surrey, BC, Canada, 2011.

[32] D. Gautam, V. Vittal, and T. Harbour, "Impact of Increased Penetration of DFIG-Based Wind Turbine Generators on Transient and Small Signal Stability of Power Systems," IEEE Transactions on Power Systems, vol. 24, no. 3, pp. 1426-1434, 2009.

[33] P. N. Papadopoulos and J. V. Milanovic, "Probabilistic Framework for Transient Stability Assessment of Power Systems With High Penetration of Renewable Generation," IEEE Transactions on Power Systems, vol. 32, no. 4, pp. 3078-3088, 2017.

[34] C. J. Tavora and O. J. M. Smith, "Characterization of Equilibrium and Stability in Power Systems," IEEE Transactions on Power Apparatus and Systems, vol. PAS-91, no. 3, pp. 1127-1130, 1972.

[35] DIgSILENT PowerFactory 15 User Manual. DIgSILENT GmbH, 2015. 